University of Vermont

UVM ScholarWorks

$10-1-2016$

\title{
Acoustic streaming and thermal instability of flow generated by ultrasound in a cylindrical container
}

\author{
Adam Green \\ University of Vermont \\ Jeffrey S. Marshall \\ University of Vermont \\ Dong $\mathrm{Ma}$ \\ University of Vermont \\ Junru Wu \\ University of Vermont
}

Follow this and additional works at: https://scholarworks.uvm.edu/cemsfac

\section{Recommended Citation}

Green A, Marshall JS, Ma D, Wu J. Acoustic streaming and thermal instability of flow generated by ultrasound in a cylindrical container. Physics of Fluids. 2016 Oct 31;28(10):104105.

This Article is brought to you for free and open access by the College of Engineering and Mathematical Sciences at UVM ScholarWorks. It has been accepted for inclusion in College of Engineering and Mathematical Sciences Faculty Publications by an authorized administrator of UVM ScholarWorks. For more information, please contact scholarworks@uvm.edu. 


\section{Acoustic streaming and thermal instability of flow generated by ultrasound in a cylindrical container}

Cite as: Phys. Fluids 28, 104105 (2016); https://doi.org/10.1063/1.4965899

Submitted: 04 February 2016 . Accepted: 10 October 2016 . Published Online: 31 October 2016

Adam Green, Jeffrey S. Marshall, Dong Ma, and Junru Wu

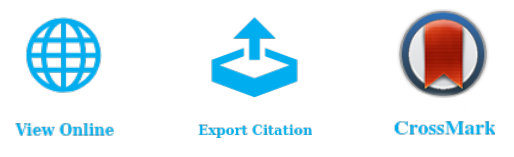

\section{ARTICLES YOU MAY BE INTERESTED IN}

Acoustic streaming, fluid mixing, and particle transport by a Gaussian ultrasound beam in a cylindrical container

Physics of Fluids 27, 103601 (2015); https://doi.org/10.1063/1.4932232

Scaling and dimensional analysis of acoustic streaming jets

Physics of Fluids 26, 093602 (2014); https://doi.org/10.1063/1.4895518

Acoustic Streaming near a Boundary

The Journal of the Acoustical Society of America 30, 329 (1958); https://

doi.org/10.1121/1.1909587

\section{Physics of Fluids GALLERY OF COVERS}




\title{
Acoustic streaming and thermal instability of flow generated by ultrasound in a cylindrical container
}

\author{
Adam Green, ${ }^{1}$ Jeffrey S. Marshall, ${ }^{1, a)}$ Dong Ma, ${ }^{2}$ and Junru $\mathrm{Wu}^{2}$ \\ ${ }^{1}$ Department of Mechanical Engineering, The University of Vermont, Burlington, \\ Vermont 05405, USA \\ ${ }^{2}$ Department of Physics, The University of Vermont, Burlington, Vermont 05405, USA
}

(Received 4 February 2016; accepted 10 October 2016; published online 31 October 2016)

\begin{abstract}
A vertically orientated ultrasonic transducer contained within a closed cylindrical Pyrex tube was used to study the acoustic streaming flow within a cylindrical container. A particle-image velocimetry (PIV) system incorporating fluorescent $1.5 \mu \mathrm{m}$ seeding particles suspended in a mixture of diethyl-phthalate and ethanol, whose optical index was matched to that of Pyrex, was used to allow for undistorted PIV imaging within the Pyrex tube. Temperature on the end-wall surface and acoustic pressure within the cylinder were measured for different end-wall materials. Variables considered included acoustic absorption and reflection coefficients, ultrasound intensity, container height, and thermal properties of the end-wall material. It was observed that a quasi-steady flow field driven by acoustic streaming is rapidly established within the container, which is typically dominated by a stationary vortex ring with downward flow along the ring axis. After sufficient time this quasi-stationary flow exhibits a thermal instability causing it to transform into a secondary flow state. Different types of secondary flow states were observed, including cases where the flow along the cylinder axis is oriented upward toward the ultrasound transducer and cases where the axial flow changes directions along the cylinder axis. Published by AIP Publishing. [http://dx.doi.org/10.1063/1.4965899]
\end{abstract}

\section{INTRODUCTION}

The acoustic streaming flow generated by an ultrasound source has been used for a wide variety of processes. A major application area involves the use of ultrasound to promote fluid mixing for biological assays and for fluids in microfluidic devices (Kuznetsova and Coakley, 2007; Monnier et al., 2000; Rahimi et al., 2014; Yang et al., 2001; and Yaralioglu et al., 2004). In small devices, turbulence cannot form due to the small Reynolds number, so acoustic streaming induced by ultrasound is often a simple and effective way to induce fluid mixing. Ultrasound is also used for micro-mixing and localized wetting of agglomerates in some food products, such as bread (Pa et al., 2014), and construction materials, such as concrete (Sayers and Grenfell, 1993 and D'Angelo et al., 1995). Ultrasound is used for the removal of particles from a surface via a process known as megasonic cleaning (Kuehn et al., 1996; Kim et al., 2009; Keswani et al., 2009; Lamminen et al., 2004; and Zhang et al., 2000). In this process, a high-intensity ultrasound wave is generated in a liquid and directed toward a contaminated surface. The acoustic pressure wave causes small cavitation bubbles to form on the surface, then detach from the surface and burst during each ultrasound cycle. The shock waves generated in the fluid from the bubble bursting causes the contamination particles to be removed from the surface, where they are suspended in the acoustic streaming flow generated by the ultrasound or by other imposed flow fields (such as a spin rinse). Ultrasound can similarly be used to manipulate particles on a surface, such as for separation or sorting operations (Devendran et al., 2014 and Lei et al., 2013) or for trapping particles in desired locations on a substrate (Wiklund et al., 2004). The acoustic streaming flow generated by ultrasound has been used to deposit small

${ }^{a)}$ Author to whom correspondence should be addressed. Electronic mail: jmarsha1 @uvm.edu. Telephone: 1 (802) 656-3826. 
liposomes from a suspension onto a substrate, either to deliver drugs to a tissue that is cancerous or otherwise diseased (Huang, 2008 and Schroeder et al., 2009) or to deliver antibiotic chemicals to a biofilm (Ma et al., 2015).

The applications mentioned above rely on the convection of particles and fluid by ultrasoundinduced acoustic streaming, together with the direct acoustic radiation force acting on particles suspended in the fluid. Acoustic streaming is categorized as either Eckart streaming, which occurs in the bulk fluid, or Rayleigh streaming, which occurs within boundary layers near a solid boundary and modifies the mean-flow no-slip condition on flow boundaries. An additional phenomenon caused by ultrasound is associated with the fact that most of the energy associated with the acoustic wave propagates through the fluid with minimal absorption, but this wave energy is quickly absorbed within the solid absorptive end-wall material when ultrasound penetrates into the solid material. The wave energy absorption causes heating of the end-wall material, resulting in a surface temperature rise which, in the presence of gravity, gives rise to a buoyancy force that can significantly alter the fluid flow.

A few papers in the recent years have examined the stability of ultrasound-induced flow fields in the presence of thermal gradients, although generally the thermal field in these papers was externally imposed and not generated by ultrasonic absorption. Flow stability for the problem of a temperature gradient imposed in the direction of acoustic propagation, and orthogonal to gravity, was examined numerically by Dridi et al. $(2008,2010)$. The acoustic streaming flow was shown to pass through a series of bifurcations as the temperature gradient and acoustic intensity were increased. The effect of acoustic streaming on the Rayleigh-Bénard thermal instability problem, in which the applied temperature gradient is normal to the dominant flow and sound propagation direction, was examined numerically by Hadid et al. (2012). The influence of thermoviscous effects, arising from the dependence of the fluid viscosity on temperature and density, on ultrasound-induced acoustic streaming in microchannels was reported by Muller and Bruus (2014).

The current paper reports on an experimental study of ultrasound-induced thermal overturning in the acoustic streaming flow. For definiteness, we examined a flow configuration in which the ultrasound field is directed downwards along the axis of a cylindrical vessel, which is closed at one end. Different types of end-wall materials were tested. The ultrasound wave impinges on the end-wall surface and is partially reflected by the end-wall-fluid interface, while the remainder of the wave passes through the interface and is absorbed by the end-wall material (Wu and Nyborg, 1992). The role of end-wall material on acoustic streaming in a closed cylindrical vessel is reported in the review by Wiklund et al. (2012), who demonstrate in an experiment with ultrasound directed downward toward the bottom of a single well in a multi-well microplate that the direction of the resulting flow along the symmetry axis of the well can change for different end-wall materials, in one case being oriented downward toward the well bottom and in another case being oriented upward toward the ultrasonic transducer. The choice of end-wall material influences the flow field both through the acoustic reflection coefficient and through temperature differences. A numerical simulation for this problem was recently presented by Marshall and Wu (2015), which was applicable for short time periods, during which the flow achieved what the authors referred to as a "quasi-steady state" that was dominated by the acoustic streaming motion. The velocity field quickly achieved a nearly constant value after the ultrasound transducer was activated, but the fluid-solid interface surface temperature at the end-wall fluid-solid interface was observed to continue to increase at a steady rate throughout the computation. While the flow along the cylinder axis was always directed downward toward the impingement surface in the Marshall and Wu (2015) computations, the simulations were performed for a relatively short time period for which the end-wall surface temperature remains below the critical value required for thermal over-turning.

The experimental method used for the study is presented in Section II, including the technique for acoustic measurements and use of particle-image velocimetry (PIV) for the velocity-field measurements. Scaling of the flow and temperature fields is discussed in Section III. Section IV presents detailed results for the acoustic streaming flow and thermal-induced transition for a case with a single cylinder height and end-wall material. The focus in this section is on understanding and documenting the transitions in the flow over time as the ultrasound heats up the end-wall material of the cylinder. Section V examines the effect of different end-wall materials and different 
cylinder heights on the acoustic streaming flow, and the resulting thermally driven flow for cases where this transition occurs. Conclusions are given in Section VI.

\section{EXPERIMENTAL METHOD}

The experiments were conducted in a cylindrical Pyrex tube with inner and outer diameters of $34 \mathrm{~mm}$ and $36 \mathrm{~mm}$, respectively, which was sealed at one end and had an ultrasound transducer of radius $b=12 \mathrm{~mm}$ placed at the other end. The sealed end is referred to as the impingement surface, since the ultrasound waves impinge on this surface and are partially absorbed into the end-wall material. Different end-wall materials were used for the impingement surface, including acrylic, alumina silica ceramic, polyurethane, and Pyrex tile, each of which was manufactured to be $25 \mathrm{~mm}$ thick. The Pyrex tube was placed in a rectangular Pyrex tank, measuring $9 \times 9 \times 7 \mathrm{~cm}$. The cylindrical vessel was placed on a layer of $6 \mathrm{~mm}$ thick polyurethane, which was then placed on the $4 \mathrm{~mm}$ thick Pyrex bottom of the rectangular tank. This arrangement ensures negligible heat transfer through the bottom of the cylindrical vessel. A schematic diagram of the experimental configuration is given in Figure 1(a), showing the inner cylindrical and outer rectangular tanks, the ultrasound transducer with beam oriented toward the cylinder end-wall material, and relevant support structures. Locations of thermocouples are indicated by open circles marked with "T." A close-up diagram of the test section is given in Figure 1(b), showing the coordinate system and various parameters characterizing the cylindrical tank and end-wall material.

The fluid flow and thermal heating were achieved using $2.25 \mathrm{MHz}$ ultrasound tone-bursts with $10 \%$ duty cycle generated by a planar transducer of $2.4 \mathrm{~cm}$ diameter (Panametrics, MA) via the combination of an arbitrary waveform function generator (33250A, Agilent, Santa Clara, CA, USA) and a $55 \mathrm{~dB}$ RF power amplifier (ENI A300, Rochester, NY, USA). Acoustic measurements were obtained from "free-field" spatial-averaged and temporal-averaged (SATA) acoustic intensities $I_{\text {SATA }}$ of $0.57,2.11,4.44$, and $6.19 \mathrm{~W} / \mathrm{cm}^{2}$, measured in a large water container using the radiation force measurement method (Wu, 1995). When the transducer is placed in the cylindrical container shown in Figure 1(b), a partial standing wave can form due to the presence of reflected waves from the end-wall material interface with the fluid, which causes the effective acoustic impedance of the container to be dependent on container depth $H$. Calculation of the intensity transmission coefficient for the cylindrical container, to obtain the fraction of the incident acoustic intensity that passes into the end-wall material, is given in the Appendix.

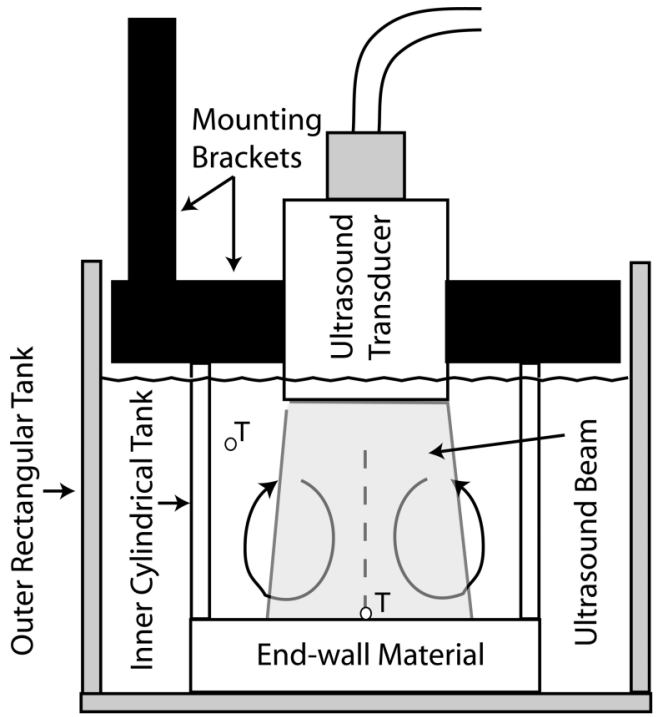

(a)

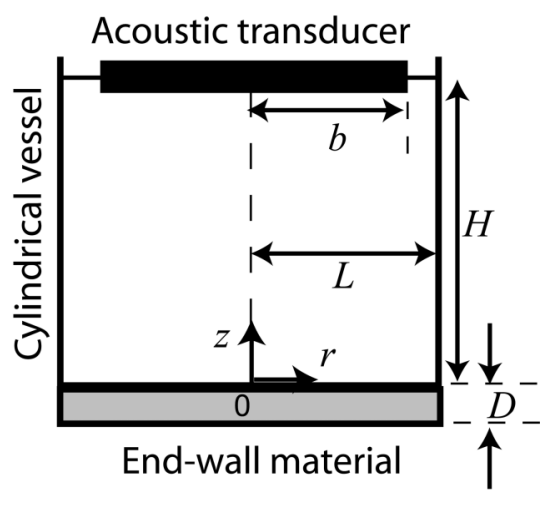

(b)

FIG. 1. Schematic diagrams of (a) the overall experimental configuration and (b) a close-up of the test section. Locations of thermocouples are indicated in (a) by open circles marked with "T." 
TABLE I. Material properties of the working fluid (82\% diethyl phthalate and 18\% 190-proof ethanol) at standard temperature and pressure and for ultrasound at $2.25 \mathrm{MHz}$ frequency.

\begin{tabular}{lccc}
\hline \hline $\begin{array}{l}\text { Density, } \rho_{F} \\
\left(\mathrm{~kg} / \mathrm{m}^{3}\right)\end{array}$ & $1053^{\mathrm{a}}$ & $\begin{array}{c}\text { Dynamic viscosity, } \mu \\
(\mathrm{Pa} \mathrm{s})\end{array}$ & $0.0049^{\mathrm{a}}$ \\
$\begin{array}{l}\text { Speed of sound, } \\
c_{F}(\mathrm{~m} / \mathrm{s})\end{array}$ & $1207-1463^{\mathrm{b}}$ & $\begin{array}{c}\text { Thermal conductivity, } \\
k_{F}(\mathrm{~W} / \mathrm{m} \mathrm{K})\end{array}$ & $0.146^{\mathrm{c}, \mathrm{d}}$ \\
$\begin{array}{l}\text { Expansion } \\
\text { coefficient, } \beta(1 / \mathrm{K})\end{array}$ & $0.00238^{\mathrm{e}}$ & $\begin{array}{c}\text { Specific heat, } \chi_{F} \\
(\mathrm{~J} / \mathrm{kg} \mathrm{K})\end{array}$ & $1813.2^{\mathrm{f}}$ \\
\hline \hline
\end{tabular}

${ }^{\mathrm{a}}$ Miller et al. (2006).

${ }^{b}$ GF Piping Systems, speed of sound in various liquids and solids. https://www.google.com/search?q=Speed+of+sound+in+various+liquids+ and + solids $\&$ ie $=$ utf $-8 \&$ oe $=$ utf -8 .

${ }^{c}$ Filippov and Novoseloca (1955).

dJamieson and Irving (1974).

${ }^{\mathrm{e}}$ Measured using the method described by Green (2016).

${ }^{\mathrm{f}}$ Chang et al. (1967).

For the fluid flow experiments, the cylindrical tube and the outer tank were filled with a mixture of $82 \%$ diethyl phthalate and 18\% 190-proof ethanol, which was selected to match the refractive index of Pyrex glass (Miller et al., 2006). Material properties of the working fluid and of the end-wall materials are listed in Tables I and II. Some of these properties were obtained from the literature and others were measured in our laboratory using the methods described by Green (2016). A listing of the different experimental cases examined in the study is given in Table III.

The fluid flow field was measured using a planar particle image velocimetry (PIV) system, consisting of a double-pulsed Nd:YAG laser (New Wave, Solo PIV II15), a digital synchronizer, a cross correlation camera with $1376 \times 1024$ pixel resolution, a 7000 NAVITAR TV zoom lens, and the TSI Insight $3 \mathrm{G}$ software for data processing. The fluid was seeded with $1.5 \mu \mathrm{m}$ mean diameter Cospheric FMO Orange Fluorescent Microsphere seeding powder, with a density of $1.3 \mathrm{~g} / \mathrm{cm}^{3}$. A number of previous investigators using PIV for the measurement of acoustic streaming flow (Campbell et al., 2000 and Barnkob et al., 2012) have pointed out the importance of using seed particles with diameter sufficiently small that the acoustic radiation force, which varies in proportion to particle volume, is negligible compared to the viscous drag force, which varies in proportion to the particle diameter. Both the theoretical estimate and experimental measures for the ratio of the acoustic radiation and viscous drag forces reported by Barnkob et al. (2012) indicate that particles of the diameter used in the current experiments follow the acoustic streaming flow accurately with the minimal influence of acoustic radiation pressure.

PIV images were taken in a vertical plane passing through the mid-point of the ultrasound transducer, with a time separation of between 2.5 and $30 \mathrm{~ms}$ between consecutive images in an

TABLE II. Properties of the materials used for the cylinder end-wall at standard temperature and pressure and for ultrasound at $2.25 \mathrm{MHz}$ frequency.

\begin{tabular}{|c|c|c|c|c|c|c|}
\hline Material & $\begin{array}{c}\text { End-wall pressure } \\
\text { reflection } \\
\text { coefficient, } R\end{array}$ & $\begin{array}{l}\text { Speed of sound, } \\
\qquad c(\mathrm{~m} / \mathrm{s})\end{array}$ & $\begin{array}{l}\text { Acoustic attenuation } \\
\text { coefficient, } \alpha_{S}(1 / \mathrm{m})\end{array}$ & $\begin{array}{c}\text { Density, } \\
\rho_{S}\left(\mathrm{~kg} / \mathrm{m}^{3}\right)\end{array}$ & $\begin{array}{c}\text { Thermal } \\
\text { conductivity, } \\
k_{S}(\mathrm{~W} / \mathrm{m} \mathrm{K})\end{array}$ & $\begin{array}{c}\text { Specific heat, } \\
\chi_{S}(\mathrm{~J} / \mathrm{kg} \mathrm{K})\end{array}$ \\
\hline Polyurethane & $0.29^{\mathrm{a}}$ & 2000 & $50.4^{\mathrm{a}}$ & 1714 & $0.02^{\mathrm{b}}$ & $1800^{c}$ \\
\hline Acrylic & $0.40^{\mathrm{a}}$ & 2890 & $29.0^{\mathrm{a}}$ & 1184 & $0.109^{d}$ & $1470^{d}$ \\
\hline Pyrex & $0.46^{\mathrm{a}}$ & 1812 & $71.0^{\mathrm{a}}$ & 2212 & $1.005^{\mathrm{c}}$ & $7500^{c}$ \\
\hline Ceramic & $0.79^{\mathrm{a}}$ & 4200 & $15.9^{\mathrm{a}}$ & 3000 & $1.08^{\mathrm{e}}$ & $1050^{\mathrm{e}}$ \\
\hline
\end{tabular}

${ }^{a}$ Measured using the method described by Green (2016).

bhttp://hyperphysics.phy-astr.gsu.edu/hbase/tables/thrcn.html.

${ }^{c}$ http://www.engineeringtoolbox.com.

dhttp://www.scribd.com/doc/8637812/Acrylic-Material-Data-from-PARSGLASS\#scribd.

${ }^{\mathrm{e}}$ Chen (2008). 
TABLE III. List of different experimental cases.

\begin{tabular}{llcc}
\hline \hline Run identifier & End-wall material & $I_{S A T A}\left(\mathrm{~W} / \mathrm{cm}^{2}\right)$ & $H(\mathrm{~mm})$ \\
\hline P1-10 & Polyurethane & 0.57 & 10 \\
P2-10 & Polyurethane & 2.11 & 10 \\
P3-10 & Polyurethane & 4.44 & 10 \\
P4-10 & Polyurethane & 6.19 & 10 \\
A1-10 & Acrylic & 0.57 & 10 \\
A2-10 & Acrylic & 2.11 & 10 \\
A3-10 & Acrylic & 4.44 & 10 \\
A4-10 & Acrylic & 6.19 & 10 \\
PR1-10 & Pyrex & 0.57 & 10 \\
PR2-10 & Pyrex & 2.11 & 10 \\
PR3-10 & Pyrex & 4.44 & 10 \\
PR4-10 & Pyrex & 6.19 & 10 \\
C1-10 & Ceramic & 0.57 & 10 \\
C2-10 & Ceramic & 2.11 & 10 \\
C3-10 & Ceramic & 4.44 & 10 \\
C4-10 & Ceramic & 6.19 & 10 \\
P2-30 & Polyurethane & 2.11 & 30 \\
P2-50 & Polyurethane & 2.11 & 50 \\
A2-30 & Acrylic & 2.11 & 30 \\
A2-50 & Acrylic & 2.11 & 50 \\
PR2-30 & Pyrex & 2.11 & 30 \\
PR2-50 & Pyrex & 2.11 & 50 \\
C2-30 & Ceramic & 2.11 & 30 \\
C2-50 & Ceramic & 2.11 & 50 \\
\hline \hline & & & \\
\hline
\end{tabular}

image pair. Vector field frames were generated at a rate of 4.8 frames/s. Image processing was performed using a double-pass image deformation method (Hart, 2000), where the first pass used an interrogation region measuring 64 pixels across and the second pass used a region measuring 32 pixels across. The processing algorithm used the initial pass to estimate the deformation that was applied during the second pass in order to enhance the measurement accuracy. Post-processing was applied to remove errant vectors and replace missing vectors. A vector was invalidated if its magnitude was more than a factor of two greater than the mean value of the surrounding 8 vectors. Missing vectors were replaced using the mean of the surrounding vectors.

The uncertainty of the PIV measurement was assessed by analyzing 18 repeated runs for a case with the polyurethane end-wall material with a distance of $10 \mathrm{~mm}$ between the end-wall and the ultrasonic emitter. An absolute velocity uncertainty was evaluated at each point of the flow field by determining the average and standard deviation of the velocity magnitude determined at a fixed time $t=12 \mathrm{~s}$ after the impulsive start, which is a sufficient time for the acoustic streaming flow to be established. Averaging the instantaneous velocity magnitude uncertainty $\Delta u_{m}$ over the flow field, we find it to be about $20 \%$ of the measured maximum velocity magnitude. To reduce the uncertainty, the velocity in many of the plots shown in the paper was averaged over some number $N$ images, such that the velocity uncertainty was reduced to $\Delta u_{m} / \sqrt{N}$.

Axisymmetry of the experimental flow field was examined by repeating an experimental time series with the PIV imaging plane rotated $90^{\circ}$ from the original imaging plane. The data in this rotated plane were obtained using an identical procedure to that described above. The data in both the original and rotated imaging planes were processed to extract flow streamlines and contour plots, as well as various other measures of the flow field. The flow was found to be highly axisymmetric, with velocity magnitude and vortex position and strength measurements in the different planes, all within experimental uncertainty of each other.

Temperature measurements were performed using a pair of OMEGA COCO-001 copper constantan thermocouples with $0.025 \mathrm{~mm}$ diameter (with Seebeck coefficient of $38 \mu \mathrm{V} /{ }^{\circ} \mathrm{C}$ ). One thermocouple was mounted using a thin film of superglue at the center of the impingement surface 
(at the interface between the end-wall material and the working fluid), and a second thermocouple was rigidly suspended within the fluid close to the side of the cylindrical container (out of the path of the ultrasonic beam, at a position $5 \mathrm{~mm}$ beneath the top fluid surface and $2.25 \mathrm{~mm}$ from the side wall). The difference in the thermocouple voltage was measured over time using a Keithly 196 System DMM, which was connected to a PC using an Agilent Technologies 82357A USB/GPIB interface. A Matlab script was used to record the change in thermocouple voltage over time on the PC. The temperature uncertainty was obtained by repeating the experiment 15 times with each of the different materials for a case with acoustic intensity $I_{S A T A}=2.11 \mathrm{~W} / \mathrm{cm}^{2}$ and height $H=50 \mathrm{~mm}$. The time-averaged standard deviation in temperature for these tests was found to vary between $3.9 \%$ and $9.1 \%$ of the temperature value.

\section{SCALING ANALYSIS}

For a continuous acoustic wave with oscillation period $\tau$, wavelength $\lambda$, and acoustic intensity vector $\mathbf{I}$ in a medium with acoustic attenuation coefficient $\alpha$, the acoustic intensity magnitude $I$ for a plane wave varies exponentially with distance $\xi$ away from the acoustic source, in proportion to $\exp (-2 \alpha \xi)$. The acoustic body force $\mathbf{F}$ and volumetric heat source function $q_{v}$ can be written for continuous wave cases where the acoustic wavelength is small compared to the transducer radius $b$ as (Nyborg, 1965 and Moudjed et al., 2014)

$$
\mathbf{F}=\frac{2 \alpha}{c} \mathbf{I}
$$

and

$$
q_{v}=-\nabla \cdot \mathbf{I}=2 \alpha I,
$$

where $c$ is the speed of sound. For a tone burst, in which the sound is emitted for a time interval of length $\Delta$ with a repetition period $t_{T B}$, a spatial average and temporal average intensity $\left(\mathbf{I}_{S A T A}\right)$ can be written as $\mathbf{I}_{S A T A}=\left(\Delta / t_{T B}\right) \mathbf{I}$, where the ratio $\Delta / t_{T B}$ is the duty cycle. In this equation, $\mathbf{I}$ is the average intensity over the time period during which the ultrasound is activated, whereas $\mathbf{I}_{S A T A}$ is the average intensity over the entire tone burst, including both times when the ultrasound is activated and times when it is off. The same Equations (1) and (2) can be used to obtain the average acoustic body force and heat source function for cases with an ultrasound tone burst simply by replacing $\mathbf{I}$ in these equations with the averaged value $\mathbf{I}_{S A T A}(\mathrm{Wu}$ and $\mathrm{Du}, 1990)$. For the ultrasound frequency $f=2.25 \mathrm{MHz}$ used in the current study, the attenuation coefficient $\alpha$ is approximately three orders of magnitude larger in the end-wall materials than it is in the working fluid (Duck, 1990). For this reason, the acoustic heating term (2) is much larger in the end-wall solid than it is in the fluid. Even though it is not significant as a heat source, acoustic attenuation in the fluid is important because it is related to the acoustic streaming body force, as indicated by expression (1).

For the velocity values observed in the current experiments, the Reynolds number is of order unity, and so the inertial and viscous terms in the Navier-Stokes equation have similar orders of magnitude. The data are observed to collapse better over the range of cases examined if we obtain the velocity scale $U_{0}$ within the acoustic streaming flow by balancing the acoustic streaming body force $\mathbf{F}$ with the viscous shear term $\mu \nabla^{2} \mathbf{u}$, where $\mu$ and $\mathbf{u}$ are the fluid viscosity and acoustic streaming velocity vector, respectively, giving

$$
U_{0}=\frac{\alpha_{F} b^{2} I_{S A T A}}{\mu c} .
$$

The Reynolds number based on the characteristic velocity scaling is defined by $\operatorname{Re}_{0} \equiv U_{0} b / v$, so from (3) we obtain

$$
\operatorname{Re}_{0}=\frac{\rho_{F} \alpha_{F} b^{3}}{c \mu^{2}} I_{S A T A} .
$$

The value of $\alpha_{F}$ for the working fluid is not recorded in the literature and is not easy to measure; however, we expect $\alpha_{F}$ to be of the same order of magnitude as the attenuation coefficient $\alpha_{W}$ of 
water. For this reason, in plotting the data it is more convenient to use an alternative characteristic velocity $U_{0}^{\prime} \equiv\left(\alpha_{W} / \alpha_{F}\right) U_{0}$.

The scale of the temperature change, $\Delta T_{0}$, is determined by balancing the acoustic energy flux $T_{I, \text { eff }} I_{S A T A}$ that passes into the end-wall surface with the convective energy flux lost from the surface, where $T_{I, e f f}$ is the effective intensity transmission coefficient given in the Appendix (Eq. (A3)). The convective energy flux is given by Newton's law of cooling as $q=h\left(T-T_{A}\right)$, where $h$ is the heat transfer coefficient at the liquid-solid interface and $T_{A}$ is the ambient temperature. Setting $\Delta T_{0}=O\left(T-T_{A}\right)$ in this balance gives the scale of the ultrasonic-induced temperature change as

$$
\Delta T_{0}=\frac{T_{I, e f f} I_{S A T A}}{h} .
$$

The heat transfer coefficient can be approximated by that for axisymmetric stagnation point flow, which is given by White (2006) as

$$
h=k_{F}(s / v)^{1 / 2} G(\operatorname{Pr}),
$$

where the fluid strain rate $s$ is assumed to be proportional to $U_{0} / b$ or $s \cong C U_{0} / b$, where $C$ is a proportionality constant, and $G(\operatorname{Pr})$ is a function of the fluid Prandtl number. While $G(\operatorname{Pr})$ must in general be obtained by a numerical solution of a system of ordinary differential equations, a curve fit to the values of $G(\operatorname{Pr})$ was given by White (2006) as

$$
G(\operatorname{Pr}) \cong 0.762 \operatorname{Pr}^{0.4} \text {. }
$$

The problem possesses multiple time scales. The fluid time scale $\tau_{F}$ is approximated by the convection time $\tau_{F, C}=b / U_{0}$. A time scale $\tau_{S}$ characteristic of heat transfer in the end-wall material can be obtained by balancing the unsteady term $\rho_{S} \chi_{S} \frac{\partial T}{\partial t}$ and the conduction term $k_{S} \nabla^{2} T$ in the temperature equation for the solid, where $\rho_{S}, \chi_{S}$, and $k_{S}$ are the density, specific heat, and thermal conductivity in the solid, respectively. We select $1 / \alpha_{S}$ as the typical length scale for thermal diffusion in the solid, instead of the much larger length scale $b$, which gives the thermal time scale in the solid as

$$
\tau_{S}=\frac{\rho_{S} \chi_{S}}{\alpha_{S}^{2} k_{S}}
$$

The ratio of the solid thermal time scale $\tau_{S}$ to the fluid time scale $\tau_{F}$ has a value ranging between 100 and 1000 for the materials used for the current experiment, indicating that the temperature of the end-wall solid responds very slowly compared to the mechanical response time of the fluid.

\section{EXPERIMENTAL RESULTS FOR THERMAL FLOW REGIME TRANSITION}

An example of the acoustic pressure field measured in a large water bath at a distance of $d=10 \mathrm{~mm}$ from the transducer face (as described in Section II) is shown in Figure 2. The acoustic intensity and frequency were $I_{S A T A}=2.11 \mathrm{~W} / \mathrm{cm}^{2}$ and $f=2.25 \mathrm{MHz}$, respectively, with the imaging plane parallel to the transducer face. Since $d=10 \mathrm{~mm} \ll \mathrm{b}^{2} / \lambda=215 \mathrm{~mm}$, where $\lambda$ is the acoustic wavelength, the measurement was made in the transducer near-field region. The acoustic pressure exhibits a beam with nearly circular shape with radius nominally equal to the transducer radius $b$. There is a ring around the outer part of the beam within which the acoustic pressure is about $15 \%$ greater than the mean value. Outside of this circle the acoustic pressure quickly decreases to a small value. The analysis and experimental results of Moudjed et al. (2015) showed that the acoustic streaming flow generated by an acoustic transducer is closely approximated by that generated by a simple parallel wave approximation, even in the transducer near field, so we do not expect the acoustic streaming flow to be sensitive to the small variation of acoustic pressure within the ultrasound beam.

An acoustic streaming flow develops within a fraction of a second after turning on the ultrasound transducer. The flow pattern for this acoustic-streaming flow is plotted in Figure 3(a) from the PIV results. The left and right hand series have different contour levels to account for the different velocity magnitudes in the acoustic streaming and thermal convection regimes. At small times, the flow is 


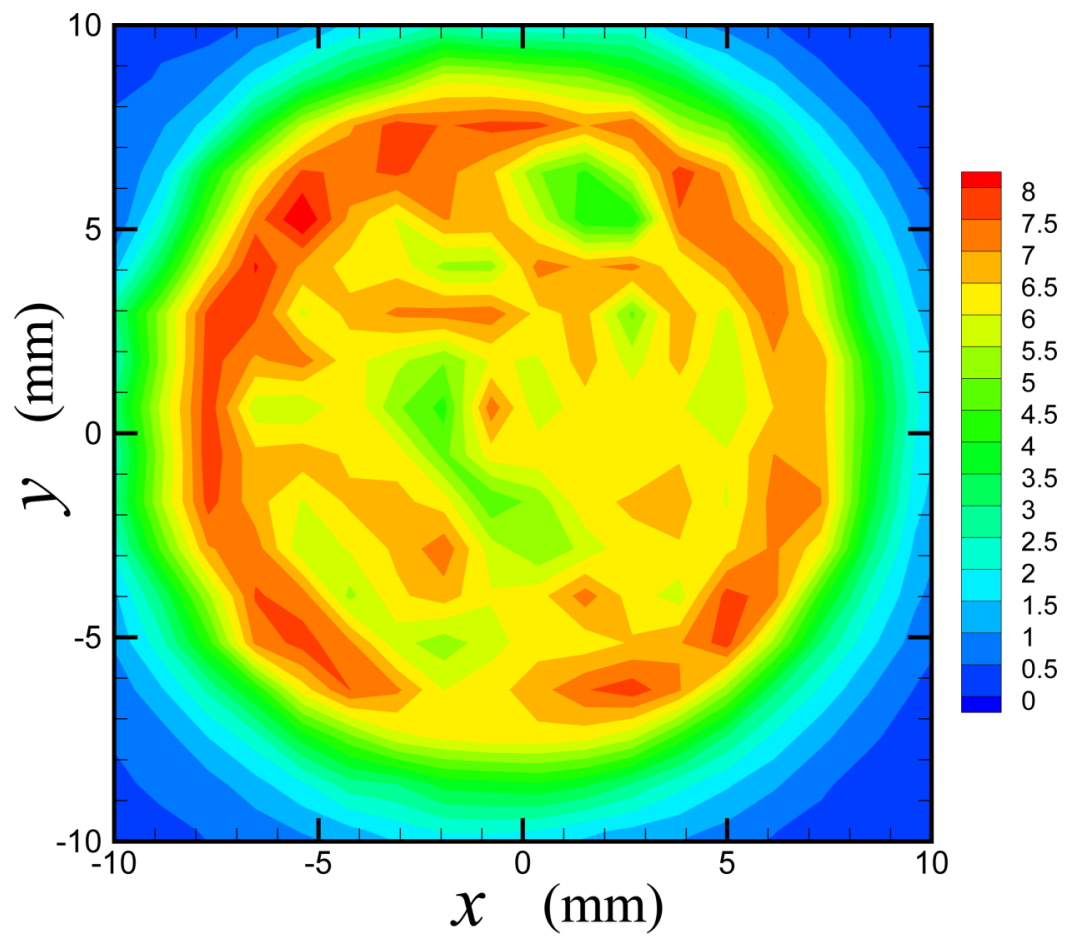

FIG. 2. Acoustic pressure (in $\mathrm{kPa}$ ) at a distance of $10 \mathrm{~mm}$ from the ultrasound transducer face.

characterized by a standing vortex ring (referred to as the acoustic streaming vortex) with the core center at $r=9 \mathrm{~mm}$ and a downward jet along the cylinder axis. A similar flow pattern is observed in the computational study of Marshall and $\mathrm{Wu}$ (2015) and in experiments reported by Wiklund et al. (2012). The velocity profile as a function of distance along the vessel mid-height line $z=H / 2$ is plotted in Figure 4(a) for four different values of the acoustic intensity. The velocity values were obtained by averaging over $N=10$ images taken at $0.015 \mathrm{~s}$ intervals, starting at a time $t=0.03 \mathrm{~s}$ after starting
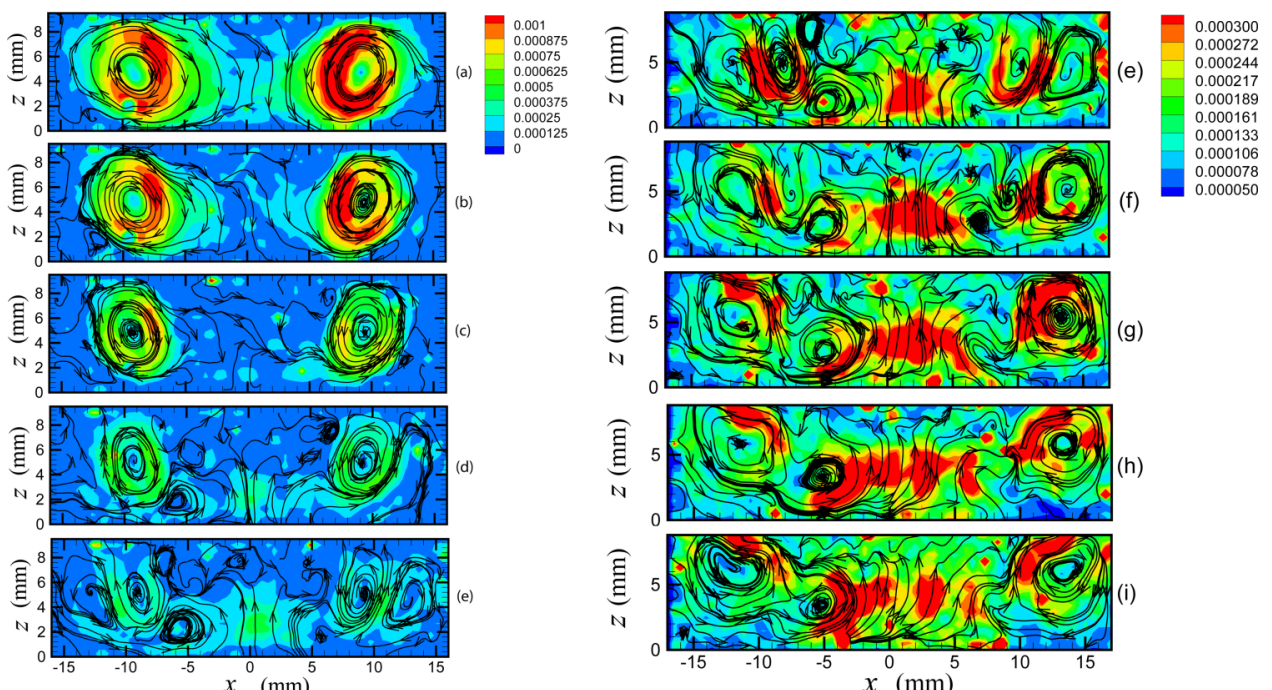

FIG. 3. Two time series showing streamlines and velocity magnitude contours (in $\mathrm{m} / \mathrm{s}$ ) for a vertical cross section of the flow for Case P2-10. Images in the first series (on the left) are obtained at times (a) $t=8.1 \mathrm{~s}$, (b) $12.2 \mathrm{~s}$, (c) $16.4 \mathrm{~s}$, (d) $20.5 \mathrm{~s}$, and (e) $24.6 \mathrm{~s}$ following start-up of the ultrasound source. Images in the second series (on the right) are for times (e) $24.6 \mathrm{~s}$, (f) $28.8 \mathrm{~s}$, (g) $32.9 \mathrm{~s}$, (h) $37.1 \mathrm{~s}$, and (i) $41.2 \mathrm{~s}$. The bottom surface of the ultrasound transducer is at the top of each image and the cylinder end-wall is at the bottom of each image. 


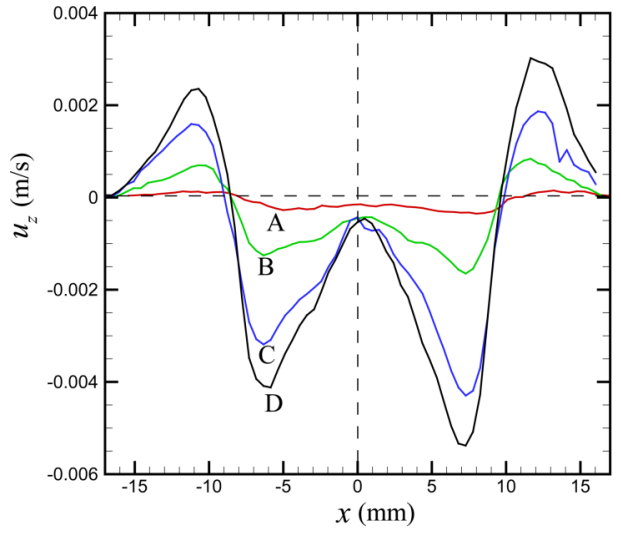

(a)

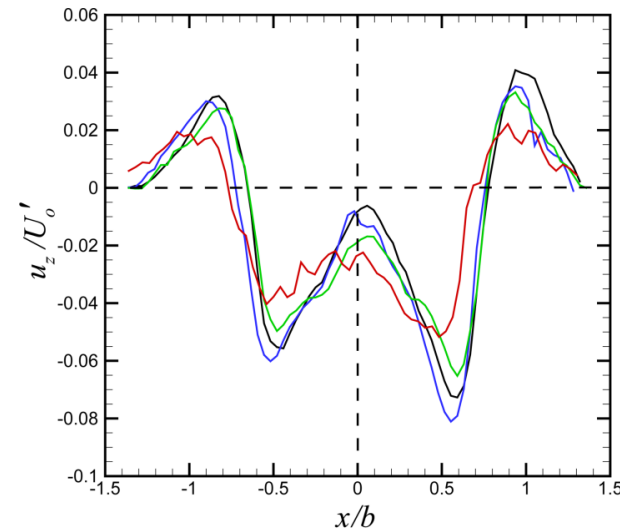

(b)

FIG. 4. Plot showing the effect of acoustic intensity $I_{S A T A}$ on the vertical velocity $u_{z}$ evaluated at the mid-plane $z=H / 2$, as a function of distance across the container: (a) vertical velocity plotted against horizontal distance, (b) dimensionless velocity ratio $u_{z} / U_{0}^{\prime}$ plotted against the dimensionless distance $x / b$. Results are for Cases P1-10 through P4-10 in Table III. Lines correspond to acoustic intensity values of $I_{S A T A}=0.57 \mathrm{~W} / \mathrm{cm}^{2}$ (A, red line), $2.11 \mathrm{~W} / \mathrm{cm}^{2}$ (B, green line), $4.44 \mathrm{~W} / \mathrm{cm}^{2}(\mathrm{C}$, blue line), and $6.19 \mathrm{~W} / \mathrm{cm}^{2}$ (D, black line).

the ultrasound. The velocity profile exhibits a peak downward velocity on the inside boundary of the vortex ring core, with a corresponding peak upward velocity on the outside boundary of the vortex ring core. The velocity along the cylinder central axis is oriented downward, but with a much smaller magnitude than the peak downward velocity. The velocity field exhibits a similar form for the different acoustic intensity values, with the expected increase in velocity magnitude as the acoustic intensity increases. Since the velocity scale $U_{0}$ in (3) is linear in the averaged acoustic intensity $I_{S A T A}$, in Fig. 4(b), we plot the dimensionless velocity ratio $u_{z} / U_{0}^{\prime}$ as a function of dimensionless distance $x / b$ for the four different intensity cases. The four velocity curves are observed to nearly collapse when scaled as shown in Fig. 4(b), thus justifying the scaling in (3).

As time progresses, the flow pattern in Fig. 3 is observed to gradually change from the acoustic streaming flow pattern, with downward velocity along the cylinder axis, to a thermal convection flow pattern, generated by ultrasonic heating of the end-wall material. Plots showing the axial velocity as a function of distance along the cylinder centerline and the shear stress on the impingement surface as a function of radius are given in Figure 5 at two different times. The velocity and shear stress values are obtained by averaging over $N=10$ images taken at $0.015 \mathrm{~s}$ intervals, where the recorded times given are the starting times of the time series. For line A the flow is in the acoustic

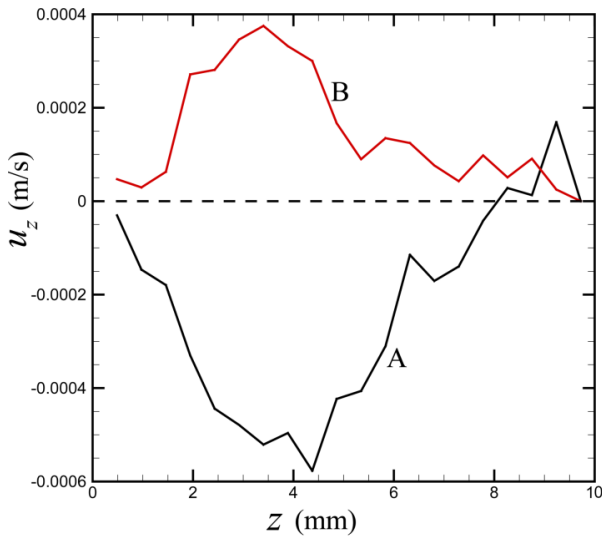

(a)

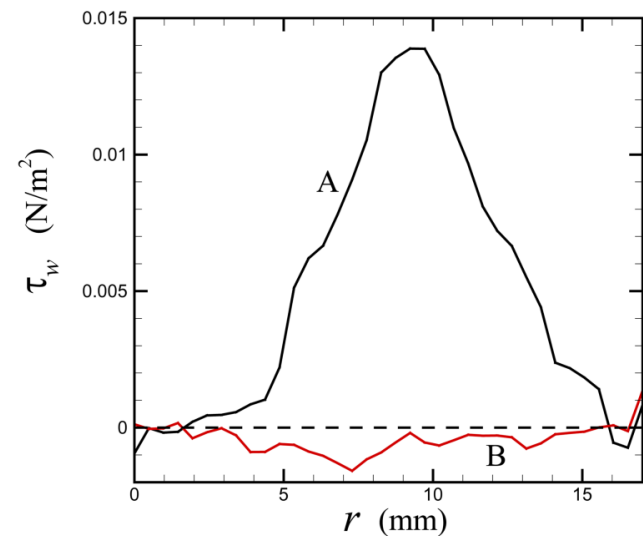

(b)

FIG. 5. Plots for the same case as Fig. 3, showing (a) variation of axial velocity along the cylinder axis and (b) variation of wall shear stress on the impingement surface with radius at times $t=2 \mathrm{~s}$ (A, black line) and $29 \mathrm{~s}$ (B, red line). 
streaming mode, whereas for line B the thermal overturning has occurred. As expected, the centerline velocity in Figure 5(a) is oriented downward (negative) in the acoustic-streaming flow and upward (positive) in the thermal convection flow. The peak value of the axial velocity magnitude occurs in both cases at approximately one-third of the distance from the impingement surface to the transducer surface, and the magnitude of the peak velocity in the thermal convection flow is about $65 \%$ of that in the acoustic streaming flow. The shear stress profile for the acoustic streaming flow exhibits a maximum value at $r=9.5 \mathrm{~mm}$, close to the position of the acoustic streaming vortex core. The wall shear stress decreases markedly after the flow transitions from acoustic streaming to thermal convection, with the maximum shear stress in the thermal convection curve only about $12 \%$ of that in the acoustic streaming curve.

The transition to a thermal convection flow is indicated by a number of changes in the flow pattern. As is evident from the contour values for velocity magnitude in Fig. 3, one change involves a gradual decrease in magnitude of the acoustic streaming flow, which is associated with a decreasing strength of the acoustic streaming vortex ring. Time variation of the strength of the acoustic streaming vortex ring is plotted in Fig. 6 for Case P2-10, where the vortex strength was computed by computing vorticity in the $r-\theta$ plane and then integrating over a circular region with radius $3.5 \mathrm{~mm}$ surrounding the vortex centroid. The decrease in strength of the acoustic streaming vortex ring, which is apparent in Fig. 6 in the time interval $t=10-30 \mathrm{~s}$, occurs because the thermal buoyancy force opposes the acoustic streaming body force within the central portion of the cylinder. A second change in the flow field occurs when the direction of the fluid velocity near the bottom of the cylinder axis changes from being oriented in a downward direction (away from the transducer, such as for curve A of Fig. 5(a)) to an upward direction (toward the transducer, such as for curve B of Fig. 5(a)). This directional change initially occurs only near the bottom of the cylinder axis, immediately above the impingement surface, but then it rapidly propagates upward so that the velocity within the entire central portion of the cylinder is oriented upward. In order to satisfy continuity, downward flows must also occur, and the resulting flow field at this transitional point adopts a more complex structure. The upward velocity near the cylinder axis opposes the direction of motion induced by the acoustic streaming vortex ring, and as a consequence multiple vortices are observed to occur and interact within the cylinder in this transitional state. A third change in the flow field occurs later in the transition process, where the acoustic streaming vortex ring fully dissipates and is replaced by a multiple vortex pattern which is primarily driven by the thermal convection flow (as shown in Figs. 3(e)-3(i)). This multiple vortex pattern is characterized by multiple upward and downward flowing jets in the spaces in-between the vortices. Once the multiple vortex pattern in the thermal convection regime has formed, it appears from Figure 3 to be approximately stationary in time.

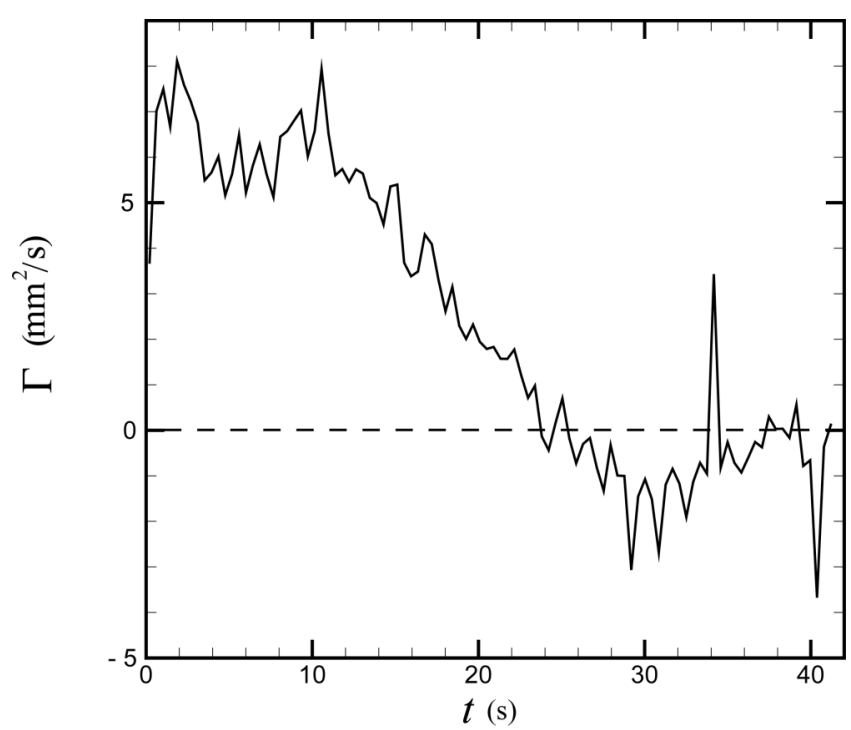

FIG. 6. Time variation of the strength of the acoustic streaming vortex ring for Case P2-10. 


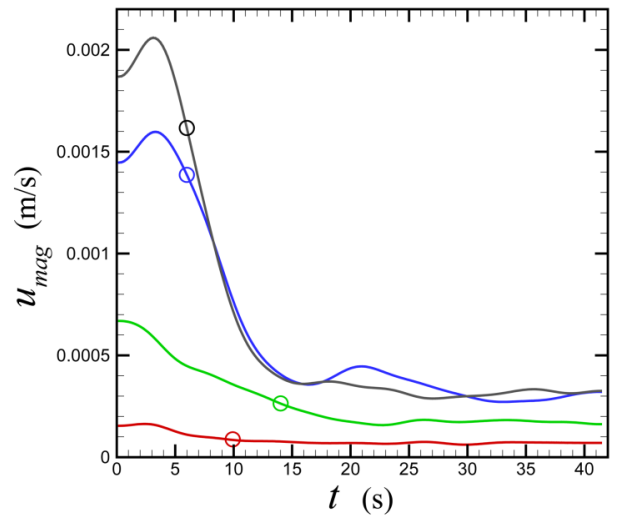

(a)

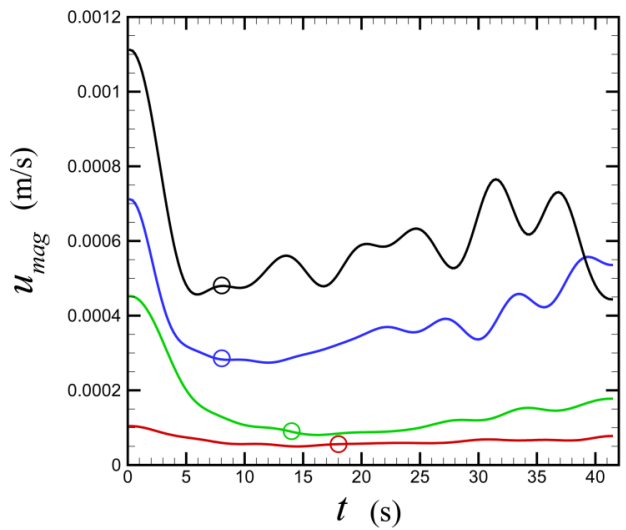

(c)

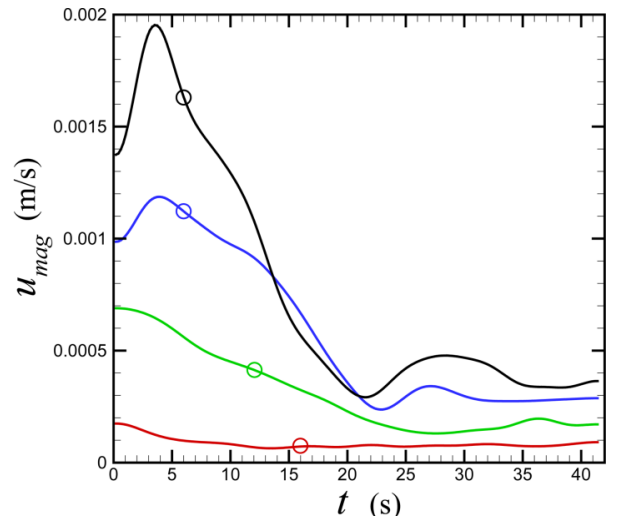

(b)

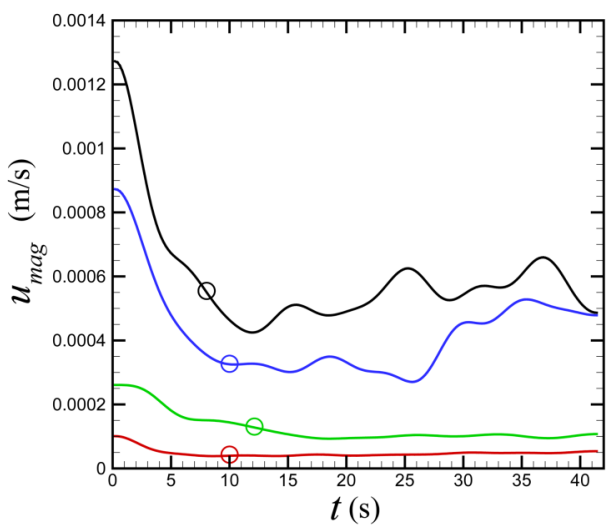

(d)

FIG. 7. Variation of mean velocity magnitude as a function of time for end-walls made of (a) polyurethane (Cases P1-10 through P4-10), (b) acrylic (Cases A1-10 through A4-10), (c) Pyrex (Cases PR1-10 through PR4-10), and (d) ceramic (Cases C1-10 through C4-10). Line colors indicate value of $I_{S A T A}$ as identified in Fig. 4. The time at which the vertical velocity on the cylinder axis changes sign is indicated by a circle.

A series of plots showing the time variation of the average velocity magnitude at four different acoustic intensity values for each of the four end-wall materials is given in Figure 7. The plots have been spectrally filtered using 20 Fourier modes to remove high-frequency noise. The time at which the axial velocity changes sign at points immediately above the impingement surface is indicated using a circle on each curve. The maximum value of velocity magnitude in the acoustic streaming flow is attained very quickly, and in some cases the time period during which the flow accelerates from zero to this maximum value could not be resolved. At short times typical of the acoustic streaming regime, the velocity is observed to increase with increase in acoustic intensity in all cases examined. All cases exhibit a significant decrease in velocity magnitude when the flow transitions from the acoustic streaming regime to the thermal convection regime. For the polyurethane and acrylic cases, the thermal effects cause a more rapid velocity magnitude decrease at the highest $I_{S A T A}$ value compared to runs at lower values of $I_{S A T A}$, which causes the velocity magnitude to sometimes reduce to a lower or nearly equal value for the $I_{S A T A}=6.19 \mathrm{~W} / \mathrm{cm}^{2}$ case as for the $I_{S A T A}=4.44 \mathrm{~W} / \mathrm{cm}^{2}$ case when the thermal regime change occurs. After the acoustic streaming vortex changes sign or breaks up into multiple vortices, the thermal convection regime is considered to be fully established and the velocity magnitude is observed for later times to be oscillatory about an approximately constant value. An exception to this is observed for the case of polyurethane end-wall material, for which the mean velocity increases slightly in time at long times.

A plot is presented in Figure 8(a) showing the time variation of the temperature difference $T-T_{A}$ between the center of the impingement surface and the bulk fluid, indicated by the two 


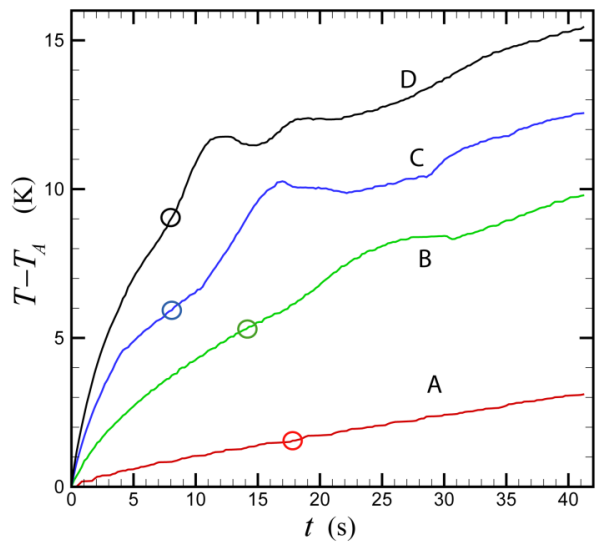

(a)

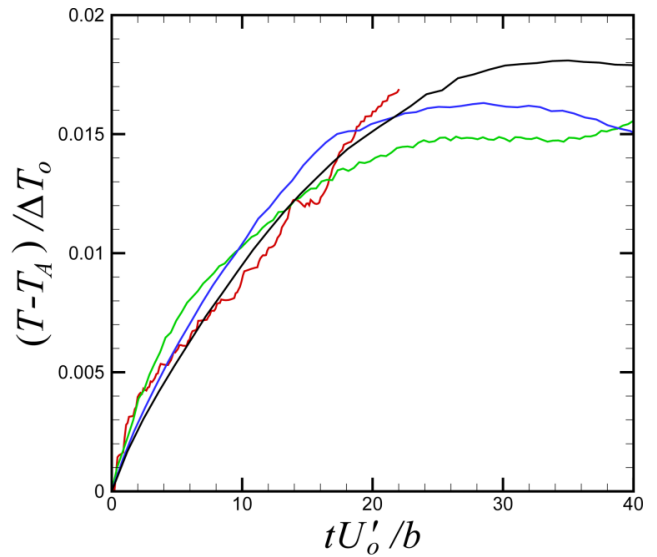

(b)

FIG. 8. Plots showing (a) temperature difference as a function of time and (b) dimensionless temperature difference as a function of dimensionless time for Cases P1-10 through P4-10. Line color indicates the value of $I_{S A T A}$ as identified in Fig. 4. The regime transition time is indicated by a circle in (a).

circles in Figure 1(a). The time at which the centerline axial velocity just above the impingement surface changes sign is indicated by a circle in each curve in Figure 8. In general, increase in the acoustic intensity causes a corresponding increase in the temperature difference. The wall temperature continues to increase throughout the experiment in accordance with the very long time scale for heat condition in the end-wall material. While we have restricted the plots to a time interval of about $40 \mathrm{~s}$ in order to focus on the transition from an acoustic-streaming to a thermal-convection dominated state, we have also performed experiments with much longer time intervals in order to examine how the system will develop as thermal equilibrium is approached. We find that the temperature difference gradually flattens out and becomes nearly independent of time by about $t=100 \mathrm{~s}$, achieving a steady state with a flow field that looks similar to that at $t=40 \mathrm{~s}$.

A collapse of the temperature data for different acoustic intensities is achieved by plotting a non-dimensional temperature $\left(T-T_{A}\right) / \Delta T_{0}$, where $T_{A}$ is the ambient temperature and $\Delta T_{0}$ is the temperature scale given in (5), against a dimensionless time $t U_{0}^{\prime} / b$, as shown for polyurethane end-wall material in Figure 8(b). This data collapse serves to validate both the temperature and time scales derived in Section III.

\section{EXPERIMENTAL RESULTS WITH DIFFERENT CYLINDER HEIGHTS}

The flow transition discussed in Sec. IV is typical of acoustic-streaming flows in this configuration for cases where the fluid depth $H$ is of the same order as the transducer radius $b$, which is typified in our experiments for the case with $H=10 \mathrm{~mm}$. All four of the materials examined exhibited qualitatively similar flow regimes for this value of $H$. As the value of $H$ increases significantly above the value of the transducer radius $b$, qualitatively different behavior can in some cases be observed, but the nature of the resulting flows is dependent on the end-wall material.

Figure 9 gives a comparison of the velocity magnitude as a function of time for cases with $H=10,30$, and $50 \mathrm{~mm}$ for the four different end-wall materials. Velocity values are averaged over 10 data sets. The velocity magnitude generally increases for larger values of $H$, particularly in the initial acoustic streaming regime. A similar trend of increasing acoustic streaming velocity with height was observed in the computations of Marshall and Wu (2015), who attributed this trend to the fact that the acoustic streaming body force acts on the fluid over a longer time period for larger values of $H$. While the cases with $H=10$ and $30 \mathrm{~mm}$ exhibit the sharp decrease in mean velocity magnitude characteristic of transition to the thermal convection regime, the velocity decrease for the cases with $H=50 \mathrm{~mm}$ is much smaller. In order to understand this behavior, velocity magnitude contour plots and streamlines at $t=49.5 \mathrm{~s}$ (near the end of the experimental run) for cases with 


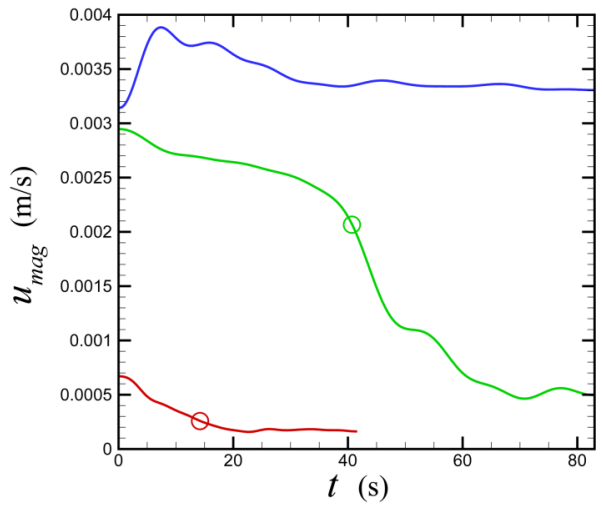

(a)

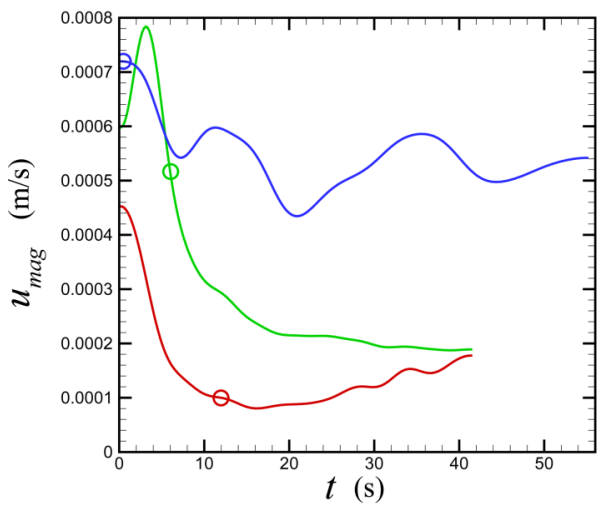

(c)

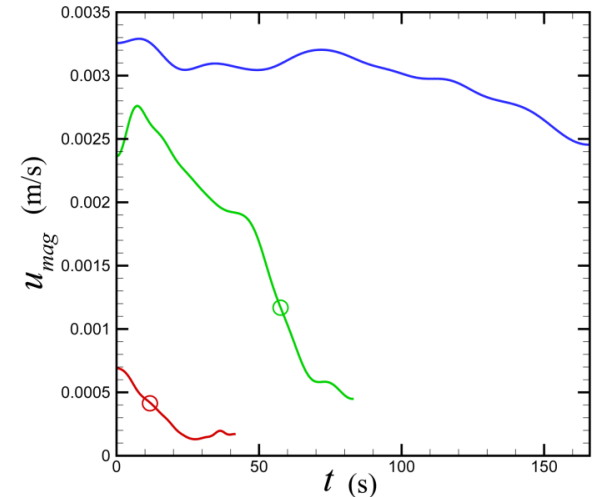

(b)

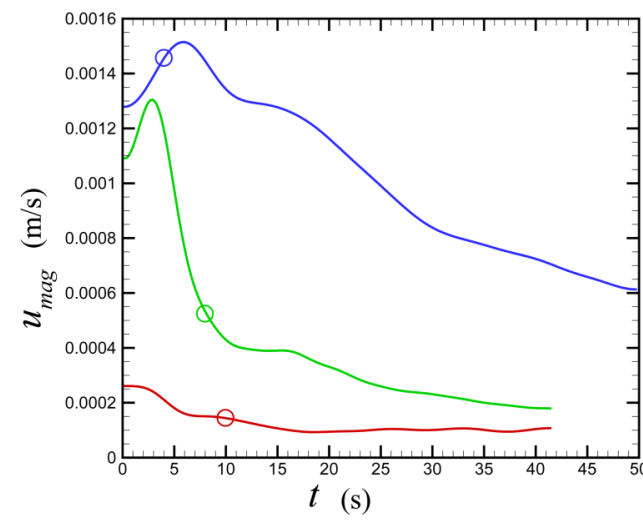

(d)

FIG. 9. Variation of mean velocity magnitude as a function of time for different end-wall materials and transducer height $H$, with ultrasound intensity $I_{S A T A}=2.11 \mathrm{~W} / \mathrm{cm}^{2}$. Lines indicate transducer height, with $H=10 \mathrm{~mm}$ (red line), $30 \mathrm{~mm}$ (green line), and $50 \mathrm{~mm}$ (blue line). The plots are for end-walls made of (a) polyurethane (P2-10, P2-30, P2-50), (b) acrylic (A2-10, A2-30, A2-50), (c) Pyrex (PR2-10, PR2-30, PR2-50), and (d) ceramic (C2-10, C2-30, C2-50). The time at which the vertical velocity on the cylinder axis changes sign is indicated by a circle.

$H=50 \mathrm{~mm}$ and intensity $I_{S A T A}=2.11 \mathrm{~W} / \mathrm{cm}^{2}$ are shown in Figure 10 for the four different end-wall materials examined. It is noted that for the polyurethane and acrylic end-wall materials having the lowest reflection coefficient (and hence higher acoustic streaming magnitude), the transition to the thermal convection driven regime has not occurred and the flow still has a downward-oriented velocity along the cylinder axis near the impingement surface as is typical of acoustic streaming flows. The ability of acoustic streaming to dominate thermal convection in these flows is associated with the larger acoustic streaming velocity magnitude for larger values of $H$. Secondly, the flow fields for both the polyurethane and acrylic end-wall materials have developed a secondary vortex loop close to the symmetry axis in the upper part of the flow, near the acoustic transducer. This secondary vortex loop has opposite sign circulation to the primary acoustic streaming vortex. This secondary vortex ring is likely formed as a result of separation of vorticity generated along the cylinder side-wall or transducer boundary layer, which is convected toward the cylinder center by the acoustic streaming flow. The secondary vortex could also be influenced by non-uniformities in acoustic beam strength, with higher sound pressure near the outer edge of the beam, as discussed in Sec. IV. At any rate, the secondary loop is very weak compared to the primary acoustic streaming vortex.

The flow field with Pyrex end-wall material exhibits a strong upward flow all along the cylinder axis, despite the fact that the acoustic streaming vortex ring (in the lower part of the cylinder) has the same direction of rotation as for the flows shown in Figures 10(a) and 10(b), and thus opposes the direction of flow along the cylinder center. A second vortex ring is observed in the upper part of 


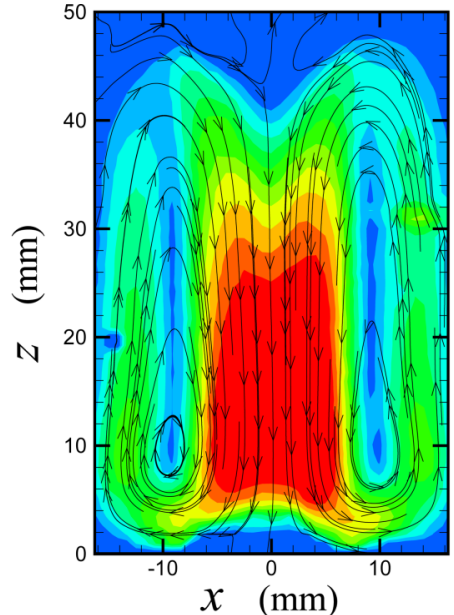

(a)

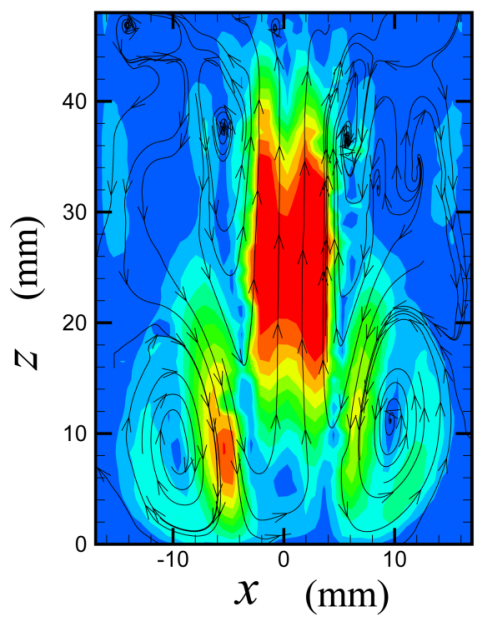

(c)

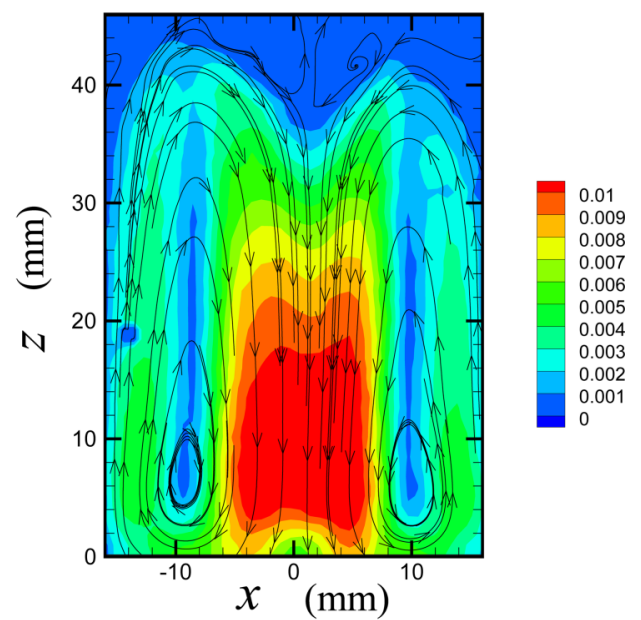

(b)

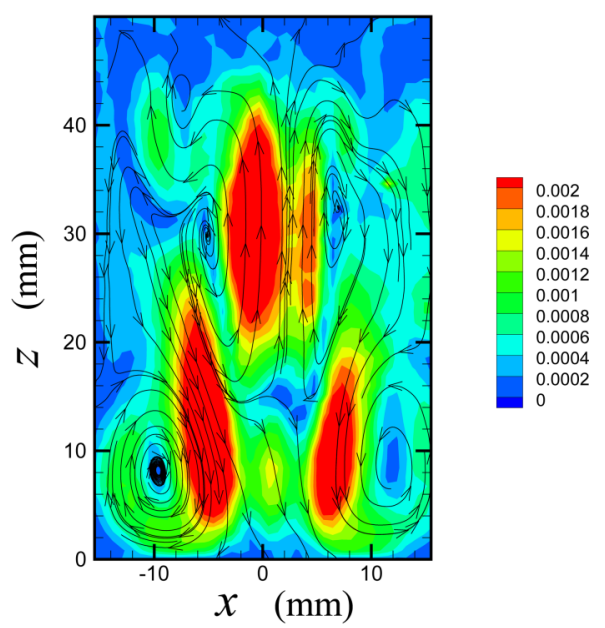

(d)

FIG. 10. Velocity magnitude contours and streamlines for cases with $H=50 \mathrm{~mm}$ at time $t=49.5 \mathrm{~s}$. The plots are made for end-walls made of (a) polyurethane (P2-50), (b) acrylic (A2-50), (c) Pyrex (PR2-50), and (d) ceramic (C2-50).

the cylinder close to the cylinder axis. This vortex ring is driven by the upward jet of fluid along the cylinder axis and the downward fluid motion on the outside of the cylinder, where the latter is forced by continuity. The case with ceramic end-wall material (Fig. 10(d)) exhibits an interesting two-cell behavior. The lower cell exhibits an acoustic streaming vortex and downward axial flow, similar to the results in Figures 10(a) and 10(b). However, the upper cell exhibits an upward axial flow with a vortex ring along the side of the axial jet, similar to the case in Figure 10(c). As time progresses the upper vortex pair extends downward, expanding in size while the lower vortex pair shrinks.

The effect of cylinder height on the radial shear stress component along the bottom wall is illustrated in Figure 11 for the polyurethane end-wall with three different values of $H$. Shear stress values are averaged over 10 repeated data sets. It is observed that the maximum radial stress increases by about an order of magnitude as $H$ increases from $10 \mathrm{~mm}$ to $50 \mathrm{~mm}$, which is roughly consistent with the scale of the velocity magnitude increase shown in Figure 9(a). For the $H=50 \mathrm{~mm}$ case, a small negative shear region was observed to form close to the symmetry axis (from about $r=0$ to $2.3 \mathrm{~mm}$ ). This negative shear region coincides with a small recirculation region that forms in this part of the flow. The velocity magnitude within this recirculation region is much smaller than within the primary recirculating region of the flow, and this small region consequently appears simply as a region with small velocity magnitude along the end-wall near the symmetry axis in the contour plot in Figure 10(a). 


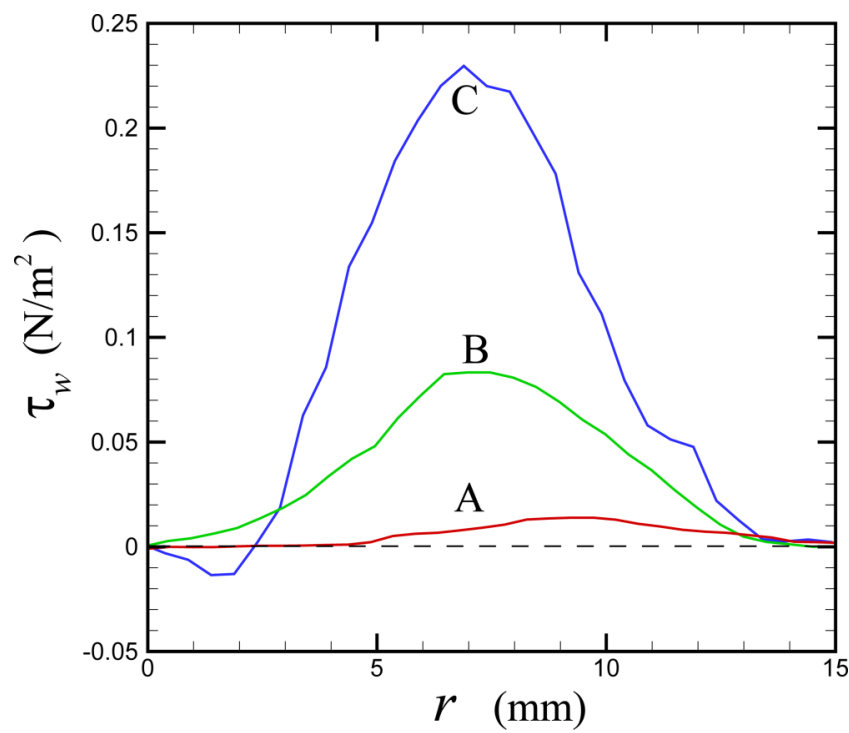

FIG. 11. Radial shear stress component along the end-wall as a function of radius for different values of $H$, showing results for Case P2-10 (A, red), Case P2-30 (B, green), and Case P2-50 (C, blue) at $t=4.4 \mathrm{~s}$.

A plot showing time variation of the temperature difference $T-T_{A}$ for the case with $H=$ $50 \mathrm{~mm}$ and $I_{S A T A}=2.11 \mathrm{~W} / \mathrm{cm}^{2}$ is shown in Figure 12(a) for the four different end-wall materials. There is a general trend for materials with lower reflection coefficients to have higher values of the temperature difference due to the fact that a larger fraction of the incident acoustic radiation passes into the end-wall material. An exception to this observation occurs for ceramic and Pyrex, however, where the much higher specific heat of Pyrex leads to a lower surface temperature even though a larger amount of energy is absorbed by the material than for ceramic.

The thermal convection regime transition time is summarized for the four different end-wall materials and the three different values of $H$ in Figure 12(b) for $I_{S A T A}=2.11 \mathrm{~W} / \mathrm{cm}^{2}$. For $H=$ $10 \mathrm{~mm}$, all of the different end-wall materials exhibit regime transition at close to the same time. However, as $H$ increases, we observe two different trends depending upon the choice of end-wall material. The cases with polyurethane and acrylic end-walls exhibit increasing transition times for

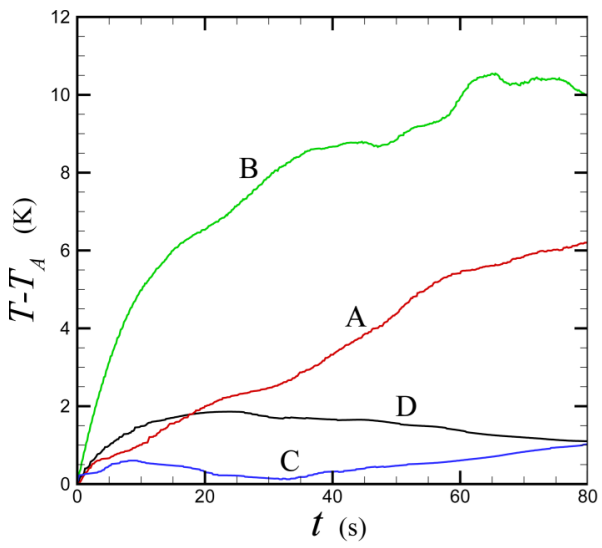

(a)

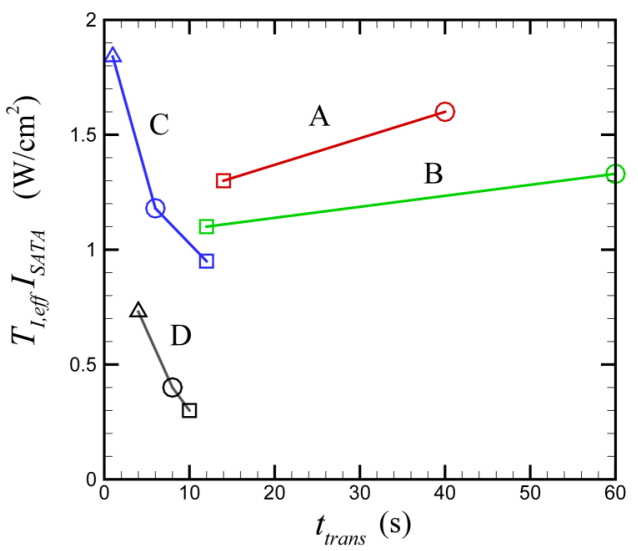

(b)

FIG. 12. Plots showing (a) the variation of temperature difference with time for cases with $H=50 \mathrm{~mm}$ (P2-50, A2-50, PR2-50, C2-50) and (b) acoustic intensity transmitted into the end-wall, $T_{I, \text { eff }} I_{S A T A}$, as a function of the transition time $t_{\text {trans }}$ to thermal convection dominated flow, with $H=10 \mathrm{~mm}$ (squares), $30 \mathrm{~mm}$ (circles), and $50 \mathrm{~mm}$ (triangles). End-wall materials include polyurethane (A, red line), acrylic (B, green line), Pyrex (C, blue line), and ceramic (D, black line). 
TABLE IV. List of the effective intensity transmission coefficients $T_{I, \text { eff }}$ for different end-wall materials as a function of container height $H$.

\begin{tabular}{lcccc}
\hline \hline $\boldsymbol{H}(\mathrm{mm}) /$ material & Polyurethane & Acrylic & Pyrex & Ceramic \\
\hline 10 & 0.6465 & 0.5135 & 0.4467 & 0.1461 \\
30 & 0.7637 & 0.6280 & 0.5544 & 0.1897 \\
50 & 1.0521 & 0.9472 & 0.8725 & 0.3447 \\
\hline \hline
\end{tabular}

$H=30 \mathrm{~mm}$ and no transition at all for $H=50 \mathrm{~mm}$. By contrast, the cases with Pyrex and ceramic end-wall materials exhibit decreasing transition times as $H$ increases. It is noted that polyurethane and acrylic have higher values of the acoustic transmission coefficient (Table IV), and hence lower reflection coefficient, than do Pyrex and ceramic. Since the acoustic body force induced by the reflected wave counteracts that from the incident wave, the velocity magnitudes are observed in Figure 9 to be significantly higher for polyurethane and acrylic than for Pyrex and ceramic. The increased value of acoustic transmission coefficient for polyurethane and acrylic also result in increased heating of the end-wall material, as evidenced by the higher temperature differences for these materials observed in Figure 12(a). The increase in acoustic streaming velocity and the increase in temperature difference for cases with higher transmission coefficient have opposing influences on the transition from the acoustic streaming to the thermal convection regime-the increased velocity increases transition time whereas the increased temperature difference decreases transition time. As $H$ is increased, Table IV indicates that the transmission coefficient is increased for all materials. The manner in which a specific material resolves the two opposing influences on the transition time depends on the nonlinear dynamics of the coupled fluid motion and heat transfer within the fluid and end-wall material.

\section{CONCLUSIONS}

The current paper reports on an experimental study of the effect of impingement surface heating on the flow induced by ultrasound radiation. For short times, we observe the formation of an acoustic streaming flow with downward velocity (oriented toward the impingement surface) along the cylinder center. A velocity scale was derived for this flow showing that the velocity magnitude increases linearly with the acoustic intensity. As the impingement surface heats up from absorption of ultrasound, a thermal buoyancy force develops that opposes the acoustic streaming body force within the fluid. The effect of the combined acoustic streaming and thermal buoyancy body forces was examined for four different end-wall materials (polyurethane, acrylic, Pyrex, and alumina silica ceramic). For all of these materials, when the container depth is of the same order or less than the transducer radius, a transition to a thermal convection driven flow is observed to occur after a finite time (on the order of 5-25 s). This transition is typified by a decrease in the strength of the acoustic streaming flow and a reversal in direction of acoustic streaming along the cylinder axis.

The velocity magnitude in the acoustic streaming flow increases with increase in depth of the cylindrical container. This observation is consistent with the fact that the distance over which the acoustic streaming body force acts on the fluid increases with $H$. The response of these deeper containers to long-time acoustic exposure is more complex than for shallower containers, and it is observed to depend on the choice of end-wall material. For instance, in experiments with cylinder depth $H=50 \mathrm{~mm}$ and transducer radius $b=12 \mathrm{~mm}$, cases with end-wall materials with small acoustic reflection coefficient have sufficiently strong acoustic streaming flow that the thermal transition was never observed to occur within the experimental period (lasting about $166 \mathrm{~s}$ ). However, in these cases a small secondary circulation cell is observed to form at the top of the cylinder, near the transducer, within which the direction of axial velocity changes from downward to upward. The thermal transition was observed to occur for cases with a larger reflection coefficient, for which the acoustic streaming flow was weaker, so that at long time either the axial velocity was oriented 
upward, away from the impingement surface, or multiple cells are formed causing the axial flow to change sign at different heights.

For applications involving removal of particles from a surface (requiring strong surface shear stress) or delivery of particles or liposomes to a surface strong (requiring rapid impingement jet flow), we have shown that a large separation distance relative to the radius of the transducer is desirable in order to increase the intensity of the acoustic streaming flow. Tuning the time duration of the acoustic emissions to ensure the presence of an acoustic-streaming driven flow (rather than thermal-convection flow) over the entire exposure period will result in optimal particle transport. The transition to thermal convection flow and the associated decrease in average fluid velocity magnitude may explain why some studies involving acoustically driven particle transport to a surface seem to achieve a saturated state after finite time, such that little further particle transport occurs after this time, as noted recently in a paper on ultrasonic delivery of liposomes to a biofilm by Ma et al. (2015).

\section{ACKNOWLEDGMENTS}

We thank the reviewers for many helpful comments. This work has been supported by NASA under Cooperative Agreement No. NNX13AD40A.

\section{APPENDIX: INTENSITY TRANSMISSION COEFFICIENT FOR THE CYLINDRICAL CONTAINER}

The acoustic intensity $I_{S A T A}$ was measured in a free-field using the radiation force method (Wu, 1995). The existence of a bottom solid plate in the cylindrical container changes the effective acoustic impedance of the transmitting medium experienced by the ultrasound transducer due to the presence of reflected waves from the end-wall surface. The effect of this impedance change can be evaluated using a one-dimensional plane-wave propagation model with three layers of media-the PZT transducer, the working fluid, and the solid end-wall. Since the acoustic wave is almost entirely absorbed within the end-wall material, reflections from the lower surface of the end-wall plate are negligible. An analysis of the acoustic transmission coefficient for this three-media model is given by Kinsler et al. (2000), which derived an expression for the intensity transmission coefficient $T_{I}$ as a function of container depth $H$ given by

$$
T_{I}=4\left[2+\left(\frac{r_{s}}{r_{t}}+\frac{r_{t}}{r_{s}}\right) \cos ^{2}(k H)+\left(\frac{r_{w}^{2}}{r_{s} r_{t}}+\frac{r_{s} r_{t}}{r_{w}^{2}}\right) \sin ^{2}(k H)\right]^{-1},
$$

where $r_{t}, r_{s}$, and $r_{w}$ are the characteristic acoustic impedances of the PZT transducer, the end-wall material, and the working fluid, respectively. The acoustic intensity transmitted into the end-wall solid is given by the intensity of the acoustic wave emanated by the transducer $\left(I_{\text {trans }}\right)$ times $T_{I}$. However, $I_{S A T A}$ is the acoustic intensity measured in the free-field, without boundary effects, when the transducer was excited under the same operating voltage. Therefore, we have

$$
I_{\text {SATA }}=I_{\text {trans }} /\left(1-R_{\text {trans }}^{2}\right),
$$

where $R_{\text {trans }}=\left(r_{t}-r_{w}\right) /\left(r_{t}+r_{w}\right)$ is the pressure reflection coefficient at the transducer surface. The acoustic intensity transmitted into the end-wall material can be written as $T_{I \text {, eff }} I_{S A T A}$, where the effective transmission coefficient $T_{I, \text { eff }}$ is given by

$$
T_{I, e f f}=T_{I} /\left(1-R_{\text {trans }}^{2}\right) .
$$

For typical PZT transducers $r_{t}=3.0 \times 10^{7} \mathrm{~kg} /\left(\mathrm{m}^{2} \mathrm{~s}\right)$, and for the working fluid used in the experiments $r_{w}=1.64 \times 10^{6} \mathrm{~kg} /\left(\mathrm{m}^{2} \mathrm{~s}\right)$. For the different end-wall materials used, the value of the ratio $r_{s} / r_{w}=1.82$ (polyurethane), 2.33 (acrylic), 2.70 (Pyrex), and 8.52 (ceramic). The value of the transducer reflection coefficient is $R_{\text {trans }}=0.906$. A listing of the effective transmission coefficients $T_{I, \text { eff }}$ for different end-wall materials and different container heights is given in Table IV. 
Barnkob, R., Augustsson, P., Laurell, T., and Bruus, H., "Acoustic radiation- and streaming-induced microparticle velocities determined by microparticle image velocimetry in an ultrasound symmetry plane," Phys. Rev. E 86, 056307 (2012).

Campbell, M., Cosgrove, J. A., Greated, C. A., Jack, S., and Rockli, D., "Review of LDA and PIV applied to the measurement of sound and acoustic streaming," Opt. Laser Technol. 32, 629-639 (2000).

Chang, S. S., Horman, J. A., and Bestul, A. B., "Heat capacities and related thermal data for diethyl phthalate crystal, glass, and liquid to 360," J. Res. Natl. Bur. Stand., Sect. A 71A(4), 293-305 (1967).

Chen, P., "Manufacturing of porous surfaces with micro-scale features for advanced heat transfer," Ph.D. dissertation, University of Michigan, Ann Arbor, 2008.

D’Angelo, R., Plona, T. J., Schwartz, L. M., and Coveney, P., “Ultrasonic measurements on hydrating cement slurries,” Adv. Cem. Based Mater. 2, 8-14 (1995).

Devendran, C., Gralinski, I., and Neild, A., "Separation of particles using acoustic streaming and radiation forces in an open microfluidic channel," Microfluid. Nanofluid. 17, 879-890 (2014).

Dridi, W., Henry, D., and Hadid, H. B., "Influence of acoustic streaming on the stability of a laterally heated three-dimensional cavity," Phys. Rev. E 77, 046311 (2008).

Dridi, W., Henry, D., and Hadid, H. B., "Stability of buoyant convection in a layer submitted to acoustic streaming," Phys. Rev. E 81, 056309 (2010).

Duck, F., Physical Properties of Tissue: A Comprehensive Reference Book (Academic Press, London, 1990).

Filippov, L. P. and Novoselova, N. S., "The thermal conductivity of solutions of normal liquid," Vestn. Moskow Univ. Ser. Fiz-Mat. 10(3), 37-40 (1955) [Chem. Abstr. 49, 11366 (1955)].

Green, A., "Structure and dynamics of two flow fields used for particle deposition onto and removal from a substrate," Ph.D dissertation, University of Vermont, Burlington, 2016.

Hadid, H. B., Dridi, W., Botton, V., Moudjed, B., and Henry, D., "Instabilities in the Rayleigh-Bénard-Eckart problem," Phys. Rev. E 86, 016312 (2012).

Hart, D., "Super-resolution PIV by recursive local-correlation," J. Visualization 3(2), 187-194 (2000).

Huang, S. L., "Liposomes in ultrasonic drug and gene delivery," Adv. Drug Delivery Rev. 60(10), 1167-1176 (2008).

Jamieson, D. T. and Irving, J. B., Report No. NEL 567, National Engineering Laboratory, Glasgow, 1974.

Keswani, M., Raghavan, S., Deymier, P., and Verhaverbeke, S., "Megasonic cleaning of wafers in electrolytic solutions: Possible role of electro-acoustic and cavitation effects," Microelectron. Eng. 86, 132-139 (2009).

Kim, W., Kim, T. H., Choi, J., and Kim, H. Y., "Mechanism of particle removal by megasonic waves," Appl. Phys. Lett. 94, 081908 (2009).

Kinsler, L. E., Frey, A. R., Coppens, A. B., and Sanders, J. V., Fundamentals of Acoustics, 4th ed. (John Wiley \& Sons, Inc., New York, 2000), pp. 153-155.

Kuehn, T. H., Kittelson, T. B., Wu, Y., and Gouk, R., "Particle removal from semiconductor wafers by megasonic cleaning," J. Aerosol Sci. 47, S427-S428 (1996).

Kuznetsova, L. A. and Coakley, W. T., "Applications of ultrasound streaming and radiation force in biosensors," Biosens. Bioelectron. 22, 1567-1577 (2007).

Lamminen, H. O., Walker, H. W., and Weavers, L. K., "Mechanisms and factors influences the ultrasonic cleaning of particlefouled ceramic membranes," J. Membr. Sci. 237, 213-223 (2004).

Lei, J., Glynne-Jones, P., and Hill, M., "Acoustic streaming in the transducer plane in ultrasonic particle manipulation devices," Lab Chip 13(11), 2133-2143 (2013).

Ma, D., Green, A. M., Willsey, G. G., Marshall, J. S., Wargo, M. J., and Wu, J. R., "Effects of acoustic streaming from moderate-intensity pulsed ultrasound for enhancing biofilm mitigation effectiveness of drug-loaded liposomes," J. Acoust. Soc. Am. 138(2), 1043-1051 (2015).

Marshall, J. S. and Wu, J. R., "Acoustic streaming, fluid mixing, and particle transport by a Gaussian ultrasound beam in a cylindrical container," Phys. Fluids 27, 103601 (2015).

Miller, P., Daielson, K., Moody, G., Slifka, A., Drexler, E., and Hertzberg, J., "Matching index of refraction using a diethyl phthalate/ethanol solution for in vitro cardiovascular models," Exp. Fluids 41, 375-381 (2006).

Monnier, H., Wilhelm, A. M., and Delmas, H., "Effects of ultrasound on micromixing in flow cell," Chem. Eng. Sci. 55, 4009-4020 (2000).

Moudjed, B., Botton, V., Henry, D., Ben Hadid, H., and Garandet, J.-P., "Scaling and dimensional analysis of acoustic streaming jets," Phys. Fluids 26, 093602 (2014).

Moudjed, B., Botton, V., Henry, D., Millet, S., Garandet, J. P., and Hadid, H. B., "Near-field acoustic streaming jet," Phys. Rev. E 91, 033011 (2015)

Muller, P. B. and Bruus, H., "Numerical study of thermoviscous effects in ultrasound-induced acoustic streaming in microchannels," Phys. Rev. E 90, 043016 (2014).

Nyborg, W. L., "Acoustic streaming," in Physical Acoustics, edited by Mason, W. P. (Academic Press, New York, 1965), Chap. II B, pp. 265-331.

Pa, N. F. C., Chin, N. L., Yusof, Y. A., and Aziz, N. A., "Power ultrasound assisted mixing effects on bread physical properties," Agric. Agric. Sci. Proc. 2, 60-66 (2014).

Rahimi, M., Aghel, B., Hatamifar, B., Akbari, M., and Alsairafi, A., "CFD modeling of mixing intensification assisted with ultrasound wave in a T-type microreactor," Chem. Eng. Process. 86, 36-46 (2014).

Sayers, C. M. and Grenfell, R. L., "Ultrasonic propagation through hydrating cements," Ultrasonics 31(3), 147-153 (1993).

Schroeder, A., Kost, J., and Barenholz, Y., "Ultrasound, liposomes, and drug delivery: Principles for using ultrasound to control the release of drugs from liposomes," Chem. Phys. Lipids 162, 1-16 (2009).

White, F. M., Viscous Fluid Flow, 3rd ed. (McGraw-Hill, New York, 2006), p. 153

Wiklund, M., Toivonen, J., Tirri, M., Hänninen, P., and Hertz, H. M., "Ultrasonic enrichment of microspheres for ultrasensitive biomedical analysis in confocal laser-scanning fluorescence detection," J. Appl. Phys. 96(2), 1242-1248 (2004). 
Wiklund, M., Green, R., and Ohlin, M., “Applications of acoustic streaming in microfluidic devices,” Lab Chip 12, 2438-2451 (2012).

Wu, J., "Calculation of acoustic radiation force generated by focused beams using the ray acoustics approach," J. Acoust. Soc. Am. 97, 2747-2750 (1995).

Wu, J. and Du, G., "Temperature elevation in tissues generated by finite-amplitude bursts of ultrasound," J. Acoust. Soc. Am. 88(3), 1562-1577 (1990).

Wu, J. and Nyborg, W.-L., "Temperature rise generated by a focused Gaussian beam in a two-layer medium,” Ultrasound Med. Biol. 18(3), 293-302 (1992).

Yang, Z., Matsumoto, S., Goto, H., Matsumoto, M., and Maeda, R., "Ultrasonic micromixer for microfluidic systems," Sens. Actuators, A 93, 266-272 (2001).

Yaralioglu, G. G., Wygant, I. O., Marentis, T. C., and Khuri-Yakub, B. T., "Ultrasonic mixing in microfluidic channels using integrated transducers," Analy. Chem. 76, 3694-3698 (2004).

Zhang, F., Busnaina, A. A., Fury, M. A., and Wang, S. Q., "The removal of deformed submicron particles from silicon wafers by spin rinse and megasonics," J. Electron. Mater. 29(2), 199-204 (2000). 